\title{
Effect of Cuff Placement on Blood Pressure Measurement in Conscious Healthy Dogs
}

\section{Carlos F Agudelo ${ }^{1 *}$, Shachar Dvir', Zeki Yilmaz ${ }^{2}$ and Meric Kocaturk ${ }^{2}$}

${ }^{1}$ Small Animal Clinic, University of Veterinary and Pharmaceutical Sciences Brno, Czech Republic

2Uludag University, Veterinary Teaching Hospital, Internal Medicine Department, 16059, Gorukle, Bursa-Turkey

\begin{abstract}
Blood pressure determination is a very helpful aid in the diagnosis and monitoring of several diseases in small animal medicine. Non-invasive methods for blood pressure measurement are currently the most rapid and practical alternative in the clinical setting, however only few studies have dealt with selecting the best cuff position for its determination. The aim of the study was to determine the best site for cuff placement (limbs and tail) during noninvasive blood pressure in healthy subjects. Arterial blood pressure was measured by use of an oscilometric system in 22 clinically healthy dogs. Five consecutive measurements were obtained from five different sites: left and right median arteries, left and right tibial cranial arteries and coccygeal artery then were compared. Significant differences were found in systolic blood pressure measured from the right hind limb. Heart rate, age, and weight were not found to have a significant influence on arterial blood pressure. We suspect that the long duration of the procedure was the culprit, leading to stress, which as a result, led to higher blood pressure on the mentioned limb. Based on our results, we concluded that all limbs as well as the tail are suitable sites for routine blood pressure determination, as long as the procedure is completed within a rational time period.
\end{abstract}

Keywords: Cuff placement; Non-invasive arterial blood pressure; Dogs; Oscillometry

\section{Introduction}

Scientific advancements in modern medicine have helped prolong significantly human life length. In the veterinary field, these achievements have been just marginal; more of them due to adequate veterinary preventive care, among others. However, it is expected that in the next decades there will be also great progresses in the field of companion animal medicine due to the tendency to suffer from conditions related to ageing, as occurs in human beings [1]. Diseases like chronic renal disease, cancer or heart disease are very common in the geriatric dog or cat [2]. A very common complication from the above mentioned circumstances is systemic hypertension, and when properly diagnosed, warrant classification and management. Systemic hypertension is of concern, because can cause injury to tissues, commonly referred to as Target-Organ Damage (TOD), which is generally a strong indication for antihypertensive therapy $[3,4]$.

Recent advancements in diagnostic techniques to determine Blood Pressure (BP) make possible to obtain rapid and accurate measurements in conscious animals. One priority for a busy practitioner and owners is to obtain quick and reliable results by using non-invasive methods. Due to the increasing accuracy, reliability, and promptness of NonInvasive Blood Pressure (NIBP) measurement systems, as well as their reasonable cost, they are now becoming a significant diagnostic tool in daily clinical practice [5]. Clinical applications range from routine screening (preventive care) through diagnosis and monitoring the course of a disease [6]. Regular follow-ups have been to gained a long-term trend-line of individual measurements [3,6]. As patients age, changes in the trend line can be used as an indication of a possible underlying disease [3]. To regard, many factors make BP determination in dogs very challenging. Since small animals have relatively small blood vessels and they possess fur, which complicates repeated measurements, $\mathrm{BP}$ determination in dogs is very challenging [7]. However, other factors such as breed, age, gender, and degree of anxiety also influence BP. Furthermore, there is a minute-to-minute spontaneous physiological change in $\mathrm{BP}$, as well as circadian changes
[4]. Due to the aforementioned reasons, during the last years, there have been attempts to increase the accuracy and reliability of NIBP techniques. The efforts to standardise have been initiated by the veterinary blood pressure society, aimed by the ACVIM, which devised a consensus statement in the form of a diagnostic protocol to minimize bias including human and devised related [3]. Although effects of body position on indirect $\mathrm{BP}$ measurements $[8,9]$ and relations between $\mathrm{BP}$ measurement techniques and selected cuff positions in dogs [10] were reported, different cuff placements and its relation with physiologic parameters using a single BP method in healthy conscious dogs are not studied yet.

The aims of the present study were to determine BP values in different body places using an Oscillometric System (OS) in conscious healthy dogs, and determine relationships between BP and heart rate (HR), body weight, age and total measurement time.

\section{Materials and Methods}

\section{Patients}

Twenty-two dogs (5 males, 17 females) between 10 months and 10 years of age $(5.3 \pm 0.98)$, weighing between $3.5-40 \mathrm{~kg}(19.3 \pm 4.6)$ participated in the study (Table 1). The subjects belonged to faculty staff and students of the University of Veterinary and Pharmaceutical Sciences Brno. No requirements, other than being clinically normal were established.

*Corresponding author: Carlos F Agudelo, Assistant Professor, University of Veterinary Medicine and Pharmaceutical Sciences Brno, Small Animal Clinic Palackeho tr. 1946/1, 61242 Brno, Brno, 61242, Czech Republic, Tel: 420-608178-019; E-mail: cagudelo@vfu.cz

Received June 16, 2016; Accepted July 19, 2016; Published July 25, 2016

Citation: Agudelo CF, Dvir S, Yilmaz Z, Kocaturk M (2016) Effect of Cuff Placement on Blood Pressure Measurement in Conscious Healthy Dogs. J Vet Sci Technol 7 : 363. doi:10.4172/2157-7579.1000363

Copyright: (c) 2016 Agudelo CF, et al. This is an open-access article distributed under the terms of the Creative Commons Attribution License, which permits unrestricted use, distribution, and reproduction in any medium, provided the original author and source are credited. 


\section{Measurement of BP}

The procedure was conducted without the presence of the owners. During the procedure, only two individuals were present in the room - the investigator, which operated the equipment, and one assistant (nurse or student). A not populated room was specifically chosen for this purpose, which offered a quite environment, without interference. In there, dogs were allowed several minutes to acclimatize. Measurements of BP were acquired from the right and left median and cranial tibial arteries and coccygeal artery in both right and left lateral recumbent positions. The examined limb was contralateral to a side in which the patient was laying in order to maintain the limb at the approximate with the cuff at or close to the level of the right atrium and reduce the effect of pressure on the arteries. Commercial washable BP cuffs were used. The cuff size was determined ( $40 \%$ of the circumference of the limb) and first applied to the left forelimb (medioproximal to the carpus). The patient was minimally restrained, and once it was calm, a series of 5 measurements of BP (systolic, diastolic and mean arterial pressures) were obtained. Subsequent measurements were carried out in this order: left hind limb (above the hock), base of the tail, right forelimb and right hind limb. The sequence of measurements was maintained in all patients and the time recorded (Table 1). An automatic OS was used (Vetmon 2200, Czech Republic). Values of systolic, mean and diastolic blood pressure (SAP, MAP and DAP respectively) were displayed. The highest and lowest values were discarded, and an average of the remaining values was calculated.

\section{Statistical analysis}

Normality of all variables was confirmed using Shapiro-Wilk test. To determine differences of BP between cuff sites ANOVA test was used. A Tukey's Honestly Significant Difference (HSD) Post-Hoc tests for one-way ANOVA were used to evaluate differences between means. Pearson's correlation coefficient was used to assess associations of BP with HR, weight and age. The results in all tests were considered significant when $\mathrm{p}<0.05$

\section{Results}

A total of 550 measurements were obtained from 22 clinically normal dogs at 5 sites using an OS (Table 1). Though unrecorded, total measurement time for all BP was assessed from 35 to 90 minutes. All values distributed normally. ANOVA tests found significant differences between cuff placements for SAP $(\mathrm{p}=0.01)$ and MAP $(\mathrm{p}=0.03)$ whereas DAP did not show significant differences $(p=0.12)$. Tukey HSD PostHoc tests revealed that source of difference in SAP (consequently in MAP) among placements was due to the values obtained from the right hind limb (Figure 1).

SAP and MAP values were found to be the highest in the right cranial tibial artery $(158 \mathrm{~mm} \mathrm{Hg}$ and $120 \mathrm{~mm} \mathrm{Hg}$ respectively) (Figure 2). There were no correlations between of $B P$ with $\mathrm{HR}$, weight and age.

\section{Discussion}

In this study, BP measurements and its relation with body weight, age and measurement time were discussed based on five different cuff placements in healthy conscious dogs. Our results showed that all limbs and tail might be used for routine BP determination in dogs. There is only a handful of information in the literature and the web concerning cuff placement in veterinary patients. In general, different sources coincided that the limb one uses should be at heart level. Anecdotally on dogs, the best site for cuff placement would be over the metacarpal area and alternately, either the metatarsal area. On cats, between the elbow and the carpus, however the tail is also an acceptable site to measure

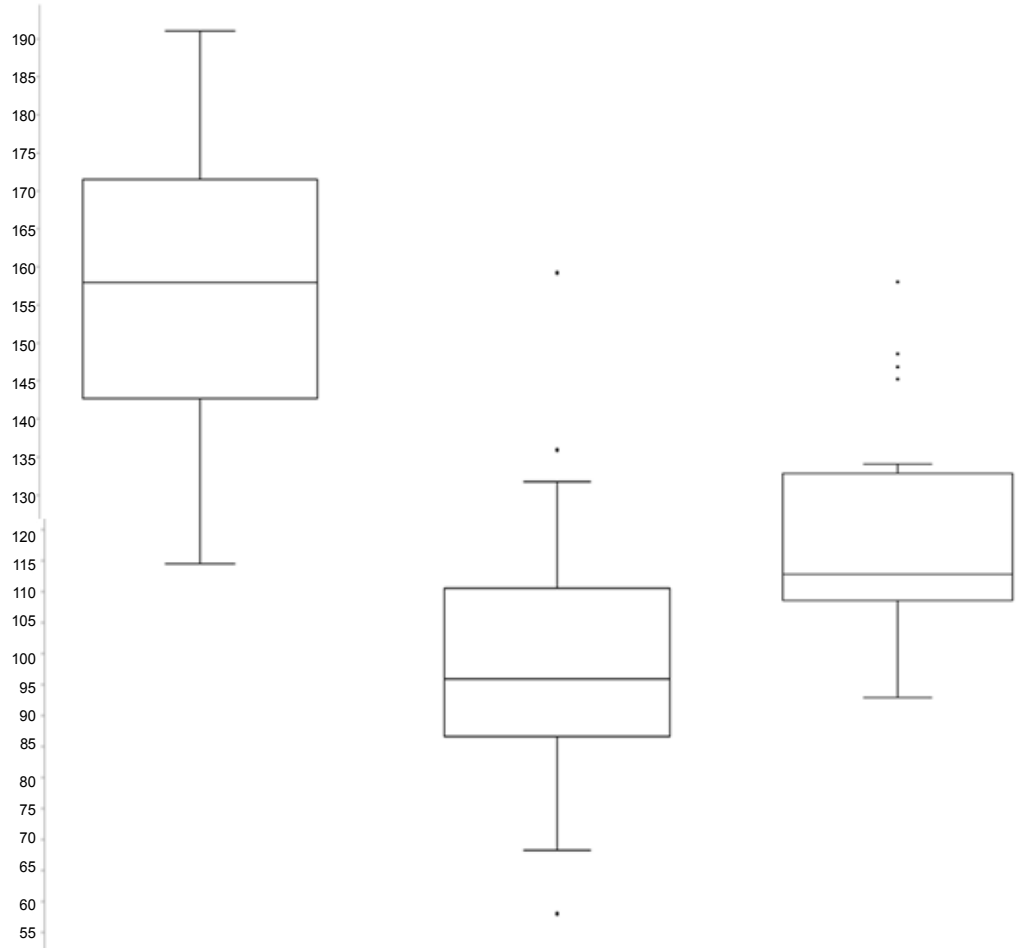

Figure 1: Results of average BP readings in 5 different cuff placements. The highest values were observed in the right hind limb at the end of whole procedure. 
Citation: Agudelo CF, Dvir S, Yilmaz Z, Kocaturk M (2016) Effect of Cuff Placement on Blood Pressure Measurement in Conscious Healthy Dogs. J Vet Sci Technol 7: 363. doi:10.4172/2157-7579.1000363

Page 3 of 4

\begin{tabular}{|c|c|c|c|c|c|c|c|c|c|c|c|c|c|c|c|c|c|}
\hline \multirow[b]{3}{*}{ Breed } & \multirow[b]{3}{*}{ Weight } & \multicolumn{6}{|c|}{ Left } & & & & \multicolumn{6}{|c|}{ Right } & \multirow[b]{3}{*}{ Duration } \\
\hline & & \multicolumn{3}{|c|}{ Forelimb } & \multicolumn{3}{|c|}{ Hind Limb } & \multicolumn{3}{|c|}{ Tail } & \multicolumn{3}{|c|}{ Forelimb } & \multicolumn{3}{|c|}{ Hind Limb } & \\
\hline & & $\mathbf{S}$ & D & M & $\mathbf{S}$ & D & M & $\mathbf{S}$ & D & M & $\mathbf{S}$ & D & M & $\mathbf{S}$ & D & M & \\
\hline Golden retriever & 34 & 131 & 80 & 105 & 155 & 71 & 90 & 127 & 69 & 92 & 148 & 86 & 101 & 138 & 96 & 113 & 60 \\
\hline $\begin{array}{c}\text { manchester } \\
\text { terrier }\end{array}$ & 10 & 151 & 97 & 114 & 169 & 108 & 140 & 144 & 87 & 110 & 147 & 95 & 118 & 185 & 125 & 145 & 86 \\
\hline GSD (Ron) & 40 & 165 & 102 & 121 & 172 & 104 & 119 & 153 & 88 & 108 & 156 & 128 & 123 & 162 & 108 & 127 & 80 \\
\hline Mixed & 12 & 95 & 71 & 82 & 162 & 124 & 142 & 147 & 95 & 113 & 144 & 115 & 124 & 169 & 129 & 143 & 97 \\
\hline Golden retriever & 25 & 121 & 59 & 79 & 121 & 40 & 83 & 97 & 53 & 68 & 126 & 73 & 92 & 149 & 57 & 103 & 37 \\
\hline Golden retriever & 30 & 133 & 61 & 82 & 119 & 43 & 74 & 117 & 57 & 80 & 155 & 79 & 106 & 135 & 78 & 99 & 35 \\
\hline mixed & 12 & 164 & 87 & 122 & 179 & 87 & 111 & 140 & 66 & 85 & 180 & 93 & 133 & 191 & 94 & 148 & 55 \\
\hline Visla & 16 & 141 & 90 & 104 & 141 & 83 & 105 & 147 & 78 & 105 & 128 & 75 & 87 & 139 & 73 & 99 & 47 \\
\hline $\begin{array}{l}\text { Wired-haired } \\
\text { visla }\end{array}$ & 20 & 145 & 92 & 107 & 126 & 70 & 88 & 151 & 101 & 116 & 127 & 94 & 105 & 176 & 95 & 108 & 122 \\
\hline WWT & 6.5 & 190 & 96 & 142 & 155 & 121 & 133 & 167 & 100 & 131 & 193 & 152 & 167 & 159 & 113 & 131 & 60 \\
\hline mixed & 32 & 147 & 78 & 90 & 160 & 78 & 112 & 134 & 61 & 99 & 131 & 74 & 95 & 140 & 89 & 110 & 60 \\
\hline mixed & 28 & 119 & 73 & 85 & 136 & 93 & 109 & 126 & 66 & 83 & 138 & 64 & 92 & 152 & 92 & 113 & 82 \\
\hline mixed & 16 & 132 & 78 & 96 & 168 & 72 & 105 & 145 & 81 & 102 & 142 & 88 & 109 & 178 & 92 & 114 & 45 \\
\hline Border Collie & 15 & 156 & 100 & 131 & 142 & 81 & 96 & 121 & 64 & 85 & 137 & 85 & 103 & 148 & 97 & 114 & 95 \\
\hline Border Collie & 15 & 117 & 71 & 86 & 129 & 64 & 83 & 134 & 84 & 105 & 135 & 72 & 96 & 142 & 87 & 112 & 79 \\
\hline Mini schnauzer & 7 & 137 & 73 & 100 & 175 & 96 & 134 & 134 & 53 & 91 & 128 & 65 & 90 & 157 & 67 & 114 & 117 \\
\hline daschhound & 8 & 190 & 121 & 133 & 203 & 104 & 161 & 123 & 57 & 93 & 145 & 66 & 89 & 162 & 88 & 114 & 42 \\
\hline Am. Staff Terrier & 25 & 148 & 113 & 126 & 147 & 112 & 133 & 179 & 121 & 149 & 164 & 127 & 146 & 162 & 108 & 129 & 99 \\
\hline GS & 25 & 121 & 62 & 77 & 144 & 103 & 117 & 113 & 73 & 99 & 119 & 66 & 96 & 121 & 79 & 95 & 30 \\
\hline mixed & 13 & 132 & 74 & 101 & 184 & 100 & 141 & 136 & 74 & 99 & 147 & 69 & 98 & 170 & 94 & 117 & 80 \\
\hline $\begin{array}{c}\text { Jack russel } \\
\text { terrier }\end{array}$ & 7 & 132 & 104 & 116 & 140 & 109 & 123 & 175 & 119 & 139 & 177 & 107 & 145 & 192 & 150 & 166 & 103 \\
\hline mixed & 3.5 & 169 & 110 & 128 & 162 & 131 & 143 & 158 & 96 & 112 & 143 & 71 & 94 & 155 & 107 & 123 & 123 \\
\hline
\end{tabular}

Table 1: Blood pressure measurements in 22 healthy dogs. Breed, weight, BP values (S: systolic; D: diastolic; M: mean), and duration of the protocol are engraved.

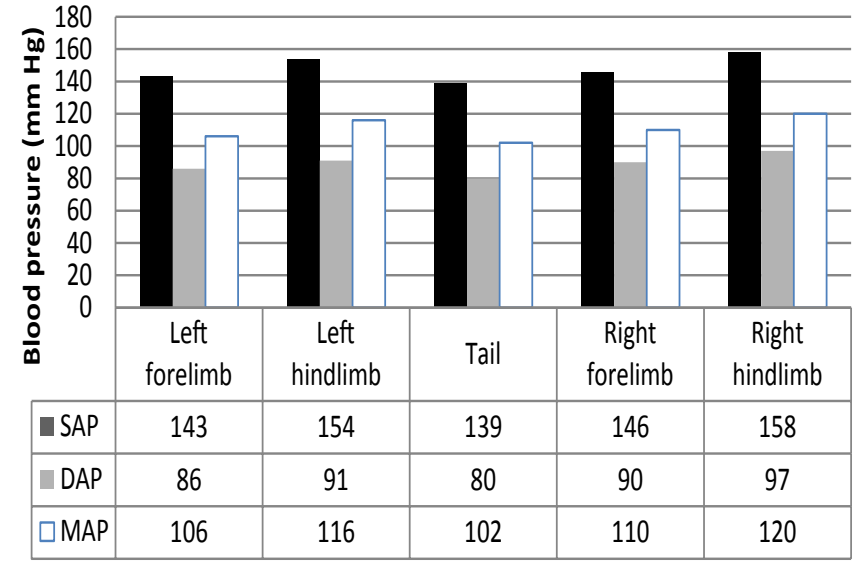

Figure 2: Results of average BP readings in 5 different cuff placements. The highest values were observed in the right hind limb at the end of whole procedure.

$\mathrm{BP}$ in both species [11]. One study determined five measurement sites (coccygeal, metacarpal, metatarsal, tibial, and median arteries) by comparing OS and ultrasonic Doppler devices with a standardized invasive radio telemetric system [12]. OS showed that coccygeal and tibial sites were the most correlated whereas Doppler determined that metatarsal site provided the strongest correlation. Another study determined reference values of BP in unsedated Irish Wolfhounds by $\mathrm{OS}$ at the coccygeal artery and the effect of body position on $\mathrm{BP}$ showing that DAP was significantly lower when measured in a standing position compared to lateral recumbence [8]. Human studies have not shown uniformity in results, where both increases and decreases in BP were observed after changes in body position $[13,14]$. These findings certainly raise the question whether there is a relationship between hypertension and posture with regards to alterations in BP.

In our study, significant changes in SAP were observed which also probably affected by directly accurate changes in the MAP. Previous research comparing simultaneous direct and indirect BP measurements suggested that SAP varies the most, either due to greater liability or greater measurement error [15]. The same conducted work also found DAP to be the least variable, both within (moment-bymoment variability) and between occasions (day-to-day variability), therefore more accurately reflecting the true BP. The differences of SAP measurements due to cuff placements (limbs and tail) were higher than that of DAP and MAP measurements. This might be explained, at least in part, by the distal pulse wave amplification phenomenon. The pulse pressure increases from the central aorta to the peripheral muscular arteries in the extremities due to reflected pulse waves and distensibility (stiffness) differences between aorta and peripheral arteries [16]. However, one should interpret carefully any result due to measurement artefacts like stress or related with operator and used devices or to the fact that SAP between occasion's decreases as more measurements are obtained. Because the above reasons, we used healthy dogs belonging to students or staff because they are get used to manipulation and to the clinical environment. These findings support once more that more emphasis should be given to DAP rather than SAP when evaluating a patient's health status using OS.

One of the main problems encountered during the procedure was the length of the OS to obtain a measurement and in some instances when movements of the dog have caused to errors. As a result, instead of completing a series of 25 measurements within the allotted 3045 minutes, in several cases it took up to 1.5 hours. Several studies have found that the traditional OS consistently underestimates BP 
in conscious dogs $[12,17,18]$. The use of high-definition oscilometric systems could refine accuracy of measurements, as it contains a 32 bit processor (compared to 8 bit processor in conventional OS) which is much less prone to errors. Undoubtedly, the length of the procedure had a significant effect on the accuracy of measurements (Table 1). First, it was difficult for some of the dogs to remain calm for such an extended period of time. Second, it was frustrating for the investigators, which could also have been sensed by the patients. Several studies have concluded that anxious dogs have higher SAP, DAP and MAP $[4,17]$.

All measurements were done in lateral recumbence in order to maintain cuff places at roughly the level of the heart. When a measurement site that is much lower than the heart, will lead to falsely elevated values and vice versa [13]. This procedure was conducted in absence of the owners. White-coat effect might have increased in some patients due to the absence of the owners, however, several of the patients were owned by the clinic's staff and students, and therefore might have been less affected. This sequence might have influenced the results in some ways, since in most cases patients became calmer during the middle part of the procedure, and then more anxious towards the end. In humans, BP machines have become increasingly affordable, allowing individuals to measure their own BP at home, thus eliminating the potential white-coat effect. If this trend trickles into the veterinary community as HDO systems becomes more affordable like other diagnostics like urine glucose, etc.; remains to be seen.

\section{Conclusion}

Significant differences were found between SAP and consequently MAP obtained from and between the limbs and the tail, when measured in lateral recumbence. However, DAP showed to be the least variable then it could be used more truthfully in reproducing the true BP. There was no significant correlation between blood pressure, heart rate, weight, and age. Although there are many limitations to this kind of study, we achieved in to determine BP under "normal" clinical conditions; therefore the variations in the measurements, as presented throughout the study are an important reminder of the challenges faced daily by clinicians in obtaining accurate and reliable data. Although cuff placement is important, if it remains the same between occasions, its importance of the technique diminishes. Furthermore, it highlights the importance of regular monitoring of BP, in order to acquire trends, which are more correlated to patient's physical condition. Further study is may be required in order to reinforce our findings.

\section{References}

1. Docksai R (2014) Extending Pet Longevity: Our Companions in Sickness and in Health. The Futurist 48: 47-51.

2. Bush BM (1989) Pharmacy and Dispensing. In: Jones's Animal Nursing. 5th edn. Lane DR (Ed.). British Small Animal Veterinary, Pp: 351-378.

3. Brown S, Atkins C, Bagley R, Carr A, Cowgill L, et al. (2007) American College of Veterinary Internal Medicine consensus statement: Guidelines for the identification, evaluation, and management of systemic hypertension in dogs and cats. J Vet Intern Med 21: 542-558
4. Egner B (2007) Blood pressure measurement-Basic principles and practical application. In: Essential facts of blood pressure in dogs and cats: a reference guide. 4th edn. Egner B, Carr A, Brown S (Eds.). Life learn, Inc., Pp: 1-14.

5. Erhardt W, Henke J, Carr A (2007) Techniques. In: Essential facts of blood pressure in dogs and cats: a reference guide. 4th edn. Egner B, Carr A, Brown $\mathrm{S}$ (Eds.). Life Learn Inc., Pp: 34-58

6. Egner B, Erhardt W, Henke J, Carr A (2007) Indications for Blood Pressure Measurements. In: Essential facts of blood pressure in dogs and cats: reference guide. 4th edn. Egner B, Carr A, Brown S (Eds.). Life Learn Inc. Pp: 15-31.

7. Remillard RL, Ross JN, Eddy JB (1991) Variance of indirect blood pressure measurements and prevalence of hypertension in clinically normal dogs. Am $J$ Vet Res 52: 561-565.

8. Bright JM, Dentino M (2002) Indirect arterial blood pressure measurement in nonsedated Irish wolfhounds: reference values for the breed. J Am Anim Hosp Assoc 38: 521-526.

9. Rondeau DA, Mackalonis ME, Hess RS (2013) Effect of body position on indirect measurement of systolic arterial blood pressure in dogs. J Am Vet Med Assoc 242: 1523-1527.

10. Garofalo NA, Teixeira Neto FJ, Alvaides RK, de Oliveira FA, Pignaton W, et al (2012) Agreement between direct, oscillometric and Doppler ultrasound blood pressures using three different cuff positions in anesthetized dogs. Vet Anaesth Analg 39: 324-334

11. Turner SM (2005) Veterinary Ophthalmology: A Manual for Nurses and Technicians. Elsevier Health Sciences. 205 pages

12. Haberman CE, Kang CW, Morgan JD, Brown SA (2006) Evaluation of oscillometric and Doppler ultrasonic methods of indirect blood pressure estimation in conscious dogs. Can J Vet Res 70: 211-217.

13. Netea RT, Smits $P$, Lenders JW, Thien T (1998) Does it matter whether blood pressure measurements are taken with subjects sitting or supine? J Hypertens 16: $263-268$.

14. Lu LC, Wei TM, Li S, Ye XL, Zeng CL, et al. (2008) Differences in blood pressure readings between supine and sitting postions in hypertensive patients. Acta Cardiol 63: 707-711.

15. Bodey AR, Mitchel AR (1997) Longitudinal studies of reproducibility and variability of indirect (oscillometric) blood pressure measurements in dogs: evidence for tracking. Res Vet Sci 63: 15-21.

16. Avolio AP, Van Bortel LM, Boutouyrie P, Cockcroft JR, McEniery CM, et al (2009) Role of pulse pressure amplification in arterial hypertension: experts opinion and review of the data. Hypertension 54: 375-383.

17. Bodey AR, Michell AR, Bovee KC, Buranakurl C, Garg T (1996) Comparison of direct and indirect (oscillometric) measurement of arterial blood pressure in conscious dogs. Res Vet Sci 61: 17-21.

18. Chalifoux A, Dallaire A, Blais D, Lariviere N, Pelletier N (1985) Evaluation of the arterial blood pressure of dogs by two noninvasive methods. Can J Comp Med 49: 419-423. 